\title{
A NOTE ON HOLONOMY
}

ANDREW P. WHITMAN AND LAWRENCE CONLON

1. Introduction. Let $M$ be a connected paracompact $C^{\infty}$ manifold, $K$ a Lie group, and $\pi: P \rightarrow M$ a principal $K$-bundle. In this paper we attack the problem of finding the possible holonomy groups for connections in $P$. By [9, Chapter II, Theorems 7.1 and 8.2] this is equivalent to finding the possible reductions of $P$.

Let $\Omega$ be the $C^{\infty}$ loop space on $M$ (parametrized on $[0,1]$ ). Any connection $\omega$ on $P$ defines a map $h_{\omega}: \Omega \rightarrow K$. The homotopy properties of this map place restrictions on the subgroups of $K$ which can occur as holonomy groups for connections in $P$. This material is treated in $\$ 2$ along with other topological preliminaries.

In $\$ 3$ we consider the principal $K$-bundle $G \rightarrow G / K$ for $(G, K)$ a compact symmetric pair. Using Nomizu's canonical connection $\omega$, we compute $h_{\omega}$ in lower dimensions. This enables us, in $\S 4$, to obtain significant restrictions on holonomy for certain of the $(G, K)$.

The more usual approach to this sort of problem is by consideration of the characteristic ring of the bundle. We do not claim essential superiority for the present method, but we do believe that it is intuitively more enlightening and, for the cases treated here, computationally simpler than an approach through characteristic classes.

We wish to thank the referee for a number of helpful comments.

2. Topological preliminaries. The following remarks summarize material fairly well known to topologists, although we do not know an explicit reference.

Topologize $\Omega$ by demanding that convergence mean uniform convergence of the tangent fields. Let $p, q \in M$ be such that $u(0)=p$, $u(1)=q, \forall u \in \Omega$. Select $p_{0} \in \pi^{-1}(p)$ and $q_{0} \in \pi^{-1}(q)$. Identify $\pi^{-1}(q)$ with $K$ by corresponding $q_{0}$ to the identity $e$ of $K$. Given a connection $\omega$, define $h_{\omega}: \Omega \rightarrow K$ by $h_{\omega}(u)=v(1)$ where $v$ is the unique $\omega$-lift of $u$ with $v(0)=p_{0}$. Given two connection forms $\omega_{t}, t=0,1$, use the convexity of the set of all connections to define connections $\omega_{t}=t \omega_{1}$ $+(1-t) \omega_{0}, 0 \leqq t \leqq 1$. Finally define $F: \Omega \times[0,1] \rightarrow K$ by $F(u, t)=h_{\omega_{t}}(u)$.

(2.1) Proposition. $F$ is continuous. In particular, $h_{\omega_{0}}$ and $h_{\omega_{1}}$ are continuous and homotopic.

Proof. Suppose $\left(u_{n}, t_{n}\right) \rightarrow(u, t)$ in $\Omega \times[0,1]$. The $\omega_{t_{n}}$-lifts of $u_{n}$ (resp. the $\omega_{t}$-lift of $u$ ) can be written $w_{n} \cdot a_{n}$ (resp. $w \cdot a$ ) where the $w_{n}$

Received by the editors August 24, 1964. 
(resp. $w$ ) are $C^{\infty}$ curves in $P$ starting at $p_{0}$ and the $a_{n}$ (resp. $a$ ) are $C^{\infty}$ curves in $K$ starting at $e$. We may also suppose that the tangent fields $\dot{w}_{n} \rightarrow \dot{w}$ uniformly on $[0,1]$. Recall [9, pp. 69-70] that $a_{n}$ (resp. $a$ ) is the $K$-component of the integral curve to a vector field $X^{n}$ (resp. $X)$ on $K \times R$ starting at $(e, 0)$. By the construction of these fields, $X^{n} \rightarrow X$ uniformly on $K \times[0,1]$. By standard theory $F\left(u_{n}, t_{n}\right)$ $\rightarrow F(u, t$,). Q.E.D.

(2.2) CoRollaRy. If $H \subset K$ is the holonomy group for some connection in $P$, then $h_{\omega}$ deforms to a map factoring through $H$.

Remark that in homology $h_{\omega^{*}}$ is a well defined homomorphism of Pontryagin rings. We do not use this fact, but it seems potentially interesting.

Let $G$ be a compact connected and simply connected Lie group, $K$ and $H$ closed connected subgroups. $H$ acts on $G / K$ in a natural way. The following lemma will be of use in $\$ 4$.

(2.3) Lemma. If $H^{*}(G / K ; R)$ is an exterior algebra on odd dimensional generators and if $H$ is transitive on $G / K$, then the natural projection $\pi: H \rightarrow G / K$ induces an injection in real cohomology.

Proof. $\pi_{0}(H)=0$ and $\pi_{1}(G / K)=0$, hence $\pi_{0}(H \cap K)=0$ and the spectral sequence of $H \cap K \rightarrow H \rightarrow G / K$ is well behaved. We must show that the transgression $t$ is zero on the universally transgressive generators of $H^{*}(H \cap K)$. If not, let $x$ be such a generator of lowest dimension such that $t(x) \neq 0 . t(x)$ is even dimensional in $H^{*}(G / K)$, hence it is an expression in lower (odd) dimensional generators. By the minimality of $\operatorname{dim}(x)$ and the fact that $H^{*}(H)$ is an exterior algebra, $\pi^{*} t(x) \neq 0$, a contradiction. Q.E.D.

3. $h_{\omega}$ for a symmetric space. Let $(G, K)$ be a compact connected symmetric pair, $\beta$ the corresponding involution of $G$. $G / K$ has Riemannian structure induced by a left and right invariant metric on $G$. Set $\mathfrak{g}=\mathfrak{f} \oplus \mathfrak{m}$, the orthogonal direct sum decomposition of the Lie algebra $g$ into the +1 and -1 eigenspaces of $\beta$.

The map $\mu: G / K \rightarrow G$ defined by $\mu(x K)=x \beta\left(x^{-1}\right)$ is a $C^{\infty}$ covering map of $G / K$ onto $\exp (\mathfrak{m})$, a totally geodesic submanifold of $G$. If $K$ is the full fixed point set of $\beta, \mu$ is an imbedding into $G$. In any case, $\mu$ maps geodesics to geodesics and takes the action of $K$ on $G / K$ to the adjoint action of $K$ on $\exp (\mathfrak{m})$.

Following Bott [4], let $\nu=(p, q ; h), p$ and $q \in G / K$ and $h$ a homotopy class of paths from $p$ to $q$. There corresponds a path component $\Omega_{\nu}$ of $\Omega$. Let $M^{\nu} \subset \Omega_{\nu}$ be the set of shortest geodesics. The following 
theorem was established in [4], but we produce here a proof which sets the stage for the computation of $h_{\omega}$. We suppose $p=e K$.

(3.1) Theorem (Botr). Let $s \in M^{\nu} . M^{\nu}$ is homeomorphic to a symmetric space $H / L$, where $H$ is the connected centralizer of $\mu(q)$ in $K$, and $L \subset H$ is the centralizer of $\mu(s(t)), 0 \leqq t \leqq 1$.

Proof. $s$ being minimal, $y=s(1 / 2)$ is not conjugate to $s(0)$ along $s$. Set $\alpha=\mu(y)$. Then $H_{\alpha}$, the centralizer of $\alpha$ in $H$, has the same identity component as $L$, and $L \subset H_{\alpha}$. Since $\alpha^{2}=\mu(q), \operatorname{Ad}\left(\alpha^{2}\right) \mid H$ is the identity. If $x \in H, \beta\left(\alpha x \alpha^{-1}\right)=\alpha \alpha^{-2} x \alpha^{2} \alpha^{-1}=\alpha x \alpha^{-1}$. Since also $\alpha x \alpha^{-1}$ centralizes $\mu(q)$, it follows that $\alpha H \alpha^{-1}=H$. Thus $H / L$ is a symmetric space and, by Morse-Bott theory, $H / L \approx \mu\left(M^{\nu}\right) \approx M^{\nu}$. Q.E.D.

For $\alpha$ as above, define $\gamma: M^{\nu} \rightarrow K$, in analogy with the definition of $\mu$, by

$$
\gamma(x L)=(\operatorname{Ad}(\alpha) x) x^{-1}=\left(\operatorname{Ad}\left(\alpha^{-1}\right) x\right) x^{-1}, \quad \forall x \in H .
$$

Let $\omega$ be Nomizu's canonical connection on $\pi: G \rightarrow G / K$ [9, Note 7]. For the definition of $h_{\omega}$ take $p_{0}=e$ and $q_{0}=\alpha$. Note that since $\alpha^{2}=\mu(q)$, $\pi(\alpha)=q$.

(3.2) ThEOREM. $h_{\omega} \mid M^{\nu}=\gamma$.

Proof. Any one parameter subgroup of $G$ starting from $e$ in an $\mathfrak{m}$-direction is horizontal with respect to $\omega$. Thus for $s \in M^{\nu}, \mu(s)$ is horizontal. Furthermore, $\pi \mu s(t / 2)=s(t)$, hence the $\omega$-lift of $s$ is $\sigma(t)$ $=\mu s(t / 2), 0 \leqq t \leqq 1$. For $x \in H$, the $\omega$-lift of $x \cdot s$ is $x \sigma x^{-1}$. Thus $h_{\omega}(x \cdot s)$ $=\alpha^{-1} x \alpha x^{-1}=\gamma(x L)$. Q.E.D.

REMARKs. The map $h_{\omega}$ gives an expression for the boundary map in the homotopy sequence of a principal bundle. The map $T=h_{\omega} \mid M^{\nu}$ defined by a connection $\omega$ on a principal bundle $P \rightarrow G / K$ generalizes the characteristic map for bundles over spheres of $[12, \S \S 23,24]$. Theorem 23.2 of [12] immediately generalizes to this situation, the integer $2 n-3$ being replaced by $|\nu|-2$, where $|\nu|$ is the least positive integer that occurs as Morse index of a geodesic in $\Omega_{\nu}$. (3.2) is a generalization of $[12,23.3]$ and as such is already essentially contained in the work of Bruno Harris [6].

4. Some applications. In the following paragraphs we apply our theory to the bundles $G \rightarrow G / K$ for certain symmetric pairs $(G, K)$. We will use cohomology theory, understanding always that the coefficient ring is the real number field.

A. $(S O(2 n), U(n))$. Consider first the case $n=2 m$. Bott [4] gives $M^{\nu}=U(2 m) / S p(m)$. Let $\pi: U(2 m) \rightarrow M^{\nu}$ be the projection. Then by [6, Proposition 1] or by the theory of [1] the map

$$
\gamma^{*} \pi^{*}: H^{*}\left(M^{\nu}\right) \rightarrow H^{*}\left(M^{\nu}\right)
$$


does not vanish on the fundamental class. It follows that if $\gamma$ is homotopic to a map factoring through $H \subset U(2 m)$, then $H$ is transitive on $\boldsymbol{U}(2 m) / \boldsymbol{S} \boldsymbol{p}(m)$.

(4.1) Lemma. If $H \subset U(2 m)$ is a holonomy group for $\mathrm{SO}(4 m)$ $\rightarrow S O(4 m) / U(2 m)$, then $H$ is transitive on $U(2 m) / S p(m)$.

(4.2) Theorem. A compact holonomy group $H$ for $S O(4 m)$ $\rightarrow S O(4 m) / U(2 m)$ must be of type $A_{2 m-2} \times T^{i}, i=1,2$, or $U(2 m)$.

Proof. By (4.1) we need only find the compact connected groups $L \subset S U(2 m)$ which are transitive on $S U(2 m) / S p(m)$. (2.3) applies and enables one to prove that $H^{*}(L)$ contains an exterior algebra on generators $x_{i}$ of $\operatorname{dim} i=5,9, \cdots, 4 m-3$. These must also be in the image of $H^{*}(S U(2 m))$, so by [7, Lemma 2] one shows that the $x_{i}$ are universally transgressive. From the cohomology ring structure of compact Lie groups (cf. [11, pp. 10-14]) one deduces that $L$ must have a simple factor $\boldsymbol{A}_{2 m-2}$ or $\boldsymbol{A}_{2 m-1}$. The result on $H \subset U(2 m)$ follows easily from considerations of rank (cf. [3]). Q.E.D.

If $n=2 m+1$, it can be shown that for suitable choice of $\nu, M^{\nu}$ is again $U(2 m) / S p(m) \cdot \gamma$ factors through $U(2 m) \subset U(2 m+1)$, so the above trick can only be used for those $H \subset U(2 m)$. We proceed differently.

(4.3) Theorem. A compact holonomy group $H$ for $S O(4 m+2)$ $\rightarrow S O(4 m+2) / U(2 m+1)$ must be of type $A_{2 m-2} \times T^{i}, i=1,2,3$; $A_{2 m-2} \times A_{1} \times T^{i}, i=1,2 ; A_{2 m-1} \times T^{i}, i=1,2$; or $U(2 m+1)$.

Proof. By [6, Proposition 1] $\gamma^{*}$ is one-one on a certain subalgebra $E_{1}$ of $H^{*}(U(2 m+1))=E_{1} \otimes E_{2}$, and by $\left[7\right.$, p. 410] $E_{1}$ is an exterior algebra on generators $x_{i}, i=1,5, \cdots, 4 m-3$. By (2.2) and (3.2) $H$ has among its primitive generators elements of the above dimensions $i$. Again examining the cohomology of compact groups [11] and using the results of [3] to exclude certain possibilities, one shows that the simple factor of $H$ which explains the generator $x_{4 m-3}$ must be $\boldsymbol{A}_{2 m-2}, \boldsymbol{A}_{2 m-1}$, or $\boldsymbol{A}_{2 m}$. Because of $x_{1}$ there is also a factor $\boldsymbol{T}^{1}$. By the results of [3] it easily follows that only the asserted possibilities can occur. Q.E.D.

B. $(U(2 n), O(2 n))$. Reasoning somewhat as in [4] we obtain $M^{v}=P_{2 n-1}=O(2 n) / O(2 n-1) \times O(1)$. Consider

$$
S^{2 n-1} \underset{i}{\rightarrow} P_{2 n-1} \underset{\gamma}{\rightarrow} \mathrm{O}(2 n) \underset{j}{\rightarrow} S^{2 n-1}
$$

where $i$ is the double covering and $j$ the projection. It is well known that $j \circ \gamma \circ i$ is of degree 2. In particular, we obtain 
(4.4) Lemma. Any holonomy group

$$
H \subset O(2 n) \quad \text { for } U(2 n) \rightarrow U(2 n) / O(2 n)
$$

acts transitively (and effectively) on $S^{2 n-1}$.

(4.5) Theorem. A compact group $H$ as in (4.4) must have identity component $H_{0}$ one of the following: $S O(2 n), S U(n), U(n), S p i n(7)$ $(n=4), \operatorname{Spin}(9)(n=8)$, or, if $n=2 m, \boldsymbol{S p}(m), \boldsymbol{S p}(1) \times Z_{2} \boldsymbol{S p}(m)$.

The proof uses the well known classification of transitive effective Lie groups on spheres (cf. [11, p. 26], [10]).

A much stronger theorem is available. Arguments with homotopy groups exclude all but $S O(2 n)$ in (4.5).

(4.6) Theorem. If $n>3$, every connection on $U(2 n) \rightarrow U(2 n) / O(2 n)$ has holonomy group $O(2 n)$.

The key fact in the proof of (4.6) is that $j \circ \gamma \circ i$ is of degree 2. This implies that the projection $H_{0} \rightarrow S^{2 n-1}$ induces a map in $\pi_{2 n-1}$ with cokernel 0 or $\boldsymbol{Z}_{2}$. As an example of how to use this fact, suppose $H_{0}=S U(n)$. Then $H_{0} \cap O(2 n-1)=S U(n-1) . \pi_{2 n-2}(S U(n))=0$ by [4] and $\pi_{2 n-2}(S U(n-1))=Z_{(n-1) !}$ by [5, Theorem 5]. Thus the cokernel in question is $\boldsymbol{Z}_{(n-1) !}$, a contradiction if $n>3$.

C. $(S p(n), U(n))$. By [4] we take $M^{\nu}=U(n) / O(n)$. Consider first the case $n=2 m+1$. Let $\pi: U(n) \rightarrow M^{\nu}$ be the projection. By [1] $\gamma^{*} \pi^{*}$ does not vanish on the fundamental class. As usual, any holonomy group $H \subset U(2 m+1)$ acts transitively on $M^{\nu}$.

(4.7) Theorem. Every connection on

$$
\operatorname{Sp}(2 m+1) \rightarrow \operatorname{Sp}(2 m+1) / U(2 m+1)
$$

has holonomy group $U(2 m+1)$.

Proof. It is enough to prove that if $L \subset S U(2 m+1)$ is a closed connected subgroup transitive on $S U(2 m+1) / S O(2 m+1)$, then $L=S U(2 m+1)$. Application of (2.3) shows, in particular, that the simple generator $x_{4 m+1}$ of $H^{*}(S U(2 m+1))$ has nonzero restriction to the subgroup $L$. It follows that the projection $S U(2 m+1) \rightarrow S^{4 m+1}$ restricts to an essential map $L \rightarrow S^{4 m+1}$. By $[11$, p. 26] $L=S U(2 m+1)$. Q.E.D.

(4.8) THEOREM. The only possible compact holonomy groups for $S p(2 m) \rightarrow S p(2 m) / U(2 m)$ are of type $A_{2 m-2} \times T^{i}, i=1,2$, or $U(2 m)$.

The proof of (4.8) uses [6, Proposition 1] and the cohomology of compact groups in essentially the same ways that we have already sketched. 
D. $\left(E_{7}, E_{6} \times T^{1}\right)$. Araki $\left[2\right.$, p. 58] has computed an $M^{v}=S^{1}$ $\times\left(E_{6} / F_{4}\right)$. Again applying [6, Proposition 1] together with the remarks on p. 300 of that paper (or the theory of [1]) we obtain that any holonomy group $H=E_{6} \times T^{1}$ acts transitively on $S^{1} \times\left(E_{6} / F_{4}\right)$. $H^{*}\left(E_{6} / F_{4}\right)$ is exterior with generators $x_{9}, x_{17}$ [2, Proposition 2.5], hence (2.3) applies and our usual cohomological considerations yield

(4.9) Theorem. Any connection on $E_{7} \rightarrow E_{7} / E_{6} \times T^{1}$ has holonomy group $E_{6} \times T^{1}$.

E. $\left(E_{7}, A_{7}\right)$. To obtain $M^{v}$ select the $\alpha$ of $\S 3$ to be the nontrivial element of the center of $E_{7}$, and take $\sigma$ to be the geodesic corresponding to a suitable edge of a fundamental simplex in the root diagram of $E_{7}$. From an examination of this root system one computes $\operatorname{dim}\left(M^{\nu}\right)$ =27. $M^{v}$ is a symmetric space of $\boldsymbol{A}_{7}$, hence (cf. the table in [8, p. 354]) $M^{v}$ has universal covering $S U(8) / S p(4)$. Reasoning as in (4.2) (modulo the covering situation) we obtain

(4.10) TheOREM. The only possible compact holonomy groups for $E_{7} \rightarrow E_{7} / A_{7}$ are $A_{6}, A_{6} \times T^{1}$, or $A_{7}$.

\section{REFERENCES}

1. S. Araki, On the Brouwer degrees of some maps of compact symmetric spaces, Topology 3 (1965), 281-290.

2. Cohomology modulo 2 of the compact exceptional groups $E_{6}$ and $E_{7}, \mathrm{~J}$. Math. Osaka City Univ. (1-2) 12 (1961), 43-65.

3. A. Borel and J. De Siebenthal, Les sousgroupes fermés de rang maximum des groupes de Lie clos, Comment. Math. Helv. 23 (1949), 200-221.

4. R. Bott, The stable homotopy of the classical groups, Ann. of Math. (2) 70 (1959), 313-337.

5. - - The space of loops on a Lie group, Michigan Math. J. 5 (1958), 35-61.

6. B. Harris, Suspensions and characteristic maps for symmetric spaces, Ann. of Math. (2) 76 (1962), 295-305.

7. - On the homotopy groups of the classical groups, Ann. of Math. (2) 74 (1961), 407-413.

8. S. Helgason, Differential geometry and symmetric spaces, Academic Press, New York, 1962.

9. S. Kobayashi and K. Nomizu, Foundations of differential geometry. I, Interscience, New York, 1963.

10. D. Montgomery and H. Samelson, Transformation groups of spheres, Ann. of Math. 44 (1943), 454-470.

11. H. Samelson, Topology of Lie groups, Bull. Amer. Math. Soc. 58 (1952), 2-37.

12. N. Steenrod, The topology of fibre bundles, Princeton Univ. Press, Princeton, N. J., 1951.

GeORGETOWN UNIVERSITY AND Loyola UNIVERSity OF NEW OrLEANS 